\title{
UNIQUENESS AND NORM CONVEXITY IN THE CAUCHY PROBLEM FOR EVOLUTION EQUATIONS WITH CONVOLUTION OPERATORS
}

\author{
MONTY J. STRAUSS ${ }^{1}$
}

\begin{abstract}
Uniqueness in the Cauchy problem is shown under suitable conditions for evolution equations of the form $u_{t}(x, t)-$ $B\left(t, D_{x}\right) u(x, t)=0$, where $B$ is a pseudo-differential operator of order $k \geqq 0$ in the $x$ variables. This is proved as a corollary to a norm convexity relation. In the process of showing this, an extension to Hölder's inequality is derived.
\end{abstract}

In recent work of Nirenberg-Treves [5], [6], investigations of local solvability of linear partial and pseudo-differential equations have led to the consideration of equations of the form

$$
L u(x, t)=D_{t} u(x, t)+i B\left(x, t, D_{x}\right) u(x, t),
$$

where $(x, t) \in \Omega \times R, \Omega \subset R^{n}$, is a first order pseudo-differential operator in the $x$ variables, whose principal symbol is real, and $D_{j}=-i \partial / \partial x_{j}$, $D_{t}=-i \partial / \partial t$. Also, the work of Calderón [1], [2], [3] has shown that considerations of uniqueness for the Cauchy problem for a system or a differential equation of arbitrary order often leads to a system of equations of this form.

In this article we allow $B$ to have arbitrary nonnegative order $k$, and we consider $L=D_{t}+i B\left(t, D_{x}\right)$, where $D_{x}=\left(D_{1}, D_{2}, \cdots, D_{n}\right)$; i.e., $B$ acts as a convolution operator with respect to the $x$ variables. We remark that in [5], [6] it is shown how to reduce the more general $D_{t}+i(B+i A)$, where $B$ and $A$ both have real principal symbols, to this form in the case $k=1$ by using exponentials.

We show uniqueness in the Cauchy problem to $L u=0$ for $C^{\infty}$ solutions with compact support in the $x$ variables for each fixed $t$, provided that the principal symbol $b_{k}$ of $B$ is semidefinite and behaves in a suitable fashion

Received by the editors November 22, 1971.

AMS 1970 subject classifications. Primary 35A05, 35B30, 58G15.

Key words and phrases. Uniqueness, Cauchy problem, evolution equation, Hölder's inequality, pseudo-differential operators.

1 This material is an extension of some of the results of the author's Ph.D. thesis, written under the direction of Professor Louis Nirenberg at the Courant Institute of New York University on a National Science Foundation Graduate Fellowship.

c. American Mathematical Society 1972 
as a function of $t$. We state first a result on norm convexity:

THEOREM 1. Suppose that

$$
D_{t} u(x, t)+i B\left(t, D_{x}\right) u(x, t)=0
$$

on $\Omega \times\left[t_{0}, t_{1}\right], \Omega \subset R^{n},-\infty<t_{0}<t_{1}<\infty$, such that the principal symbol $b_{k}$ of $B$ is a real positive semidefinite pseudo-differential operator of nonnegative order $k$ and that the symbol $b$ of $B$ satisfies $b(t, \xi)=b_{k}(t, \xi)+$ $g(t, \xi)$, where $g$ is the symbol of a pseudo-differential operator of degree $j$, and such that $j$ is a nonnegative integer less than $k(g \equiv 0$ if $k=0)$. In addition, assume that, as a function of $t$, for each $\xi$ fixed, $b_{k}$ has zeros of finite order. Then, there exist positive constants $\varepsilon=\varepsilon(t, B) \leqq \frac{1}{2}$ and $C=C(\varepsilon, B)$ independent of $t$ and $u$ such that

$$
\|u(t)\| \leqq C\left[\left\|u\left(t_{0}\right)\right\|^{\varepsilon(t)}\left\|u\left(t_{1}\right)\right\|^{1-\varepsilon(t)}+\left\|u\left(t_{0}\right)\right\|^{1-\varepsilon(t)}\left\|u\left(t_{1}\right)\right\|^{\varepsilon(t)}\right]
$$

for any $t$ in $\left(t_{0}, t_{1}\right)$, where \|\|$=\|\|_{L^{2}\left(R^{n}\right)}$. Moreover, in any compact subset $\left[t_{0}+\varepsilon_{1}, t_{1}-\varepsilon_{1}\right]$ contained in $\left(t_{0}, t_{1}\right)$, a uniform $\varepsilon$ can be chosen independent of $t$, but depending on $\varepsilon_{1}$.

RemarK. If $B$ is negative semidefinite, let $t^{\prime}=-t$ and the same theorem is valid.

COROLlaRy 2. Under the above hypotheses, if $\|u\|$ vanishes at either $t=t_{0}$ or $t=t_{1}$, then $u \equiv 0$ in $\left(t_{0}, t_{1}\right)$.

This result follows immediately by applying the theorem to any given $t$ in $\left(t_{0}, t_{1}\right)$.

A similar result can be shown for diagonal systems (which may arise from higher order equations) of the form

$$
D_{t} u_{j}(x, t)+i B_{j}\left(t, D_{x}\right) u_{j}(x, t)=\sum_{l=1}^{m} G_{j l}\left(t, D_{x}\right) u_{l}(x, t), \quad j=1, \cdots, m,
$$

if each of the $B_{j}$ satisfy the hypotheses of the theorem and the $G_{j l}$ are of order $k-1$. The method of proof is identical, but technical difficulties arise if the $B_{j}$ 's are not all the same. For details, see [7].

Calderón [1], [2], [3] proved that for general first order operators $B_{j}\left(x, t, D_{x}\right)$ with symbols $b_{j}$, one has local uniqueness in the Cauchy problem for systems if the roots of the characteristic equations $\tau_{j}-$ $i b_{j}(x, t, \xi)$ (where $\xi=\left(\xi^{1}, \cdots, \xi^{n}\right)$ is the dual variable to $x$ ) satisfy the following criteria:
(a) real roots are simple,
(b) complex roots are at most double,
(c) roots do not coalesce, and
(d) imaginary parts of complex roots are bounded away from zero. 
Our result in the case $k=1$ thus allows one to eliminate hypothesis (d) for this class of equations, and hence roots may wander from the real to the complex domain.

It is to be noted that our evolution operators all satisfy property ( $\Psi$ ) of Nirenberg and Treves [5], and thus our operators are locally "solvable" (see [6]).

Proof of Theorem 1. We have $u_{t}-B\left(t, D_{x}\right) u=0$. Then

$$
\int_{R^{n}} e^{i x \cdot \xi}\left[\tilde{u}_{t}(\xi, t)-b(t, \xi) \tilde{u}(\xi, t)\right] d \xi=0
$$

where " " denotes the Fourier transform in the $x$-variables. The Fourier inversion formula then yields the ordinary differential equation $\tilde{u}_{t}(\xi, t)-$ $b_{k}(t, \xi) \tilde{u}(\xi, t)=g(t, \xi) \tilde{u}(\xi, t)$. Set $\beta(t, \xi)=\int_{t_{0}}^{t} b_{k}(s, \xi) d s$, so that $\beta(t . \xi)$ is homogeneous of degree $k$ in $|\xi|$ for $|\xi|>1$ and is the symbol of a pseudodifferential operator of order $k$ for each fixed $t$ in $\left[t_{0}, t_{1}\right]$. Moreover, $\beta\left(t_{0}, \xi\right)=0$ and $\beta(t, \xi)$ is real and monotone increasing in $t$ for $\xi$ fixed, as $b_{k} \geqq 0$ and the zeros of $b_{k}(t)$ are of finite order.

Then $(d / d t)\left(e^{-\beta(t, \xi)} \tilde{u}(\xi, t)\right)=e^{-\beta(t, \xi)} g(t, \xi) \tilde{u}(\xi, t)$, which has as solutions

and

$$
e^{-\beta(t, \xi)} \tilde{u}(\xi, t)=\tilde{u}\left(\xi, t_{0}\right)+\int_{t_{0}}^{t} e^{-\beta(s, \xi)} g(s, \xi) \tilde{u}(\xi, s) d s
$$

$$
e^{-\beta(t, \xi)} \tilde{u}(\xi, t)=e^{-\beta\left(t_{1}, \xi\right)} \tilde{u}\left(\xi, t_{1}\right)-\int_{t}^{t_{1}} e^{-\beta(s, \xi)} g(s, \xi) \tilde{u}(\xi, s) d s .
$$

We are seeking a technique that will yield uniqueness for both the forward and backward problems simultaneously, so we want to combine formulas (2) and (3) in such a way as to eliminate the exponential terms.

First, however, we must take care of the integral expressions in '(2) and (3). We set

$$
w(\xi, t)=\left|e^{-\beta(t, \xi)} \tilde{u}(\xi, t)\right|=e^{-\beta(t, \xi)}|\tilde{u}(\xi, t)| \geqq 0 .
$$

Then (2) and (3) become respectively

and

$$
w(\xi, t)=w\left(\xi, t_{0}\right)+\int_{t_{0}}^{t} g(s, \xi) w(\xi, s) d s \quad \text { for } t \in\left[t_{0}, t_{1}\right]
$$

$$
w(\xi, t)=w\left(\xi, t_{1}\right)-\int_{t}^{t_{1}} g(s, \xi) w(\xi, s) d s \quad \text { for } t \in\left[t_{0}, t_{1}\right] .
$$

On $\left[t_{0}, t_{1}\right], g(s, \xi)$ is bounded by $C_{0}|\xi|^{j}$ for $|\xi|>1$, where $C_{0}$ is a positive constant independent of $t$ and $\xi$. Hence

$$
\text { . } w(\xi, t) \leqq w\left(\xi, t_{0}\right)+C_{0}|\xi|^{j} \int_{t_{0}}^{t} w(\xi, s) d s
$$


and

$$
w(\xi, t) \leqq w\left(\xi, t_{1}\right)+C_{0}|\xi|^{j} \int_{t}^{t_{1}} w(\xi, s) d s
$$

Set

and

$$
\gamma=\gamma(\xi)=2 C_{0}|\xi|^{j}>0
$$

We claim

$$
\begin{aligned}
& M_{0}(\xi)=\max _{t \in\left[t_{0}, t_{1}\right]} \exp \left[-\gamma\left(t-t_{0}\right)\right] w(\xi, t), \\
& M_{1}(\xi)=\max _{t \in\left[t_{0}, t_{1}\right]} \exp \left[-\gamma\left(t_{1}-t\right)\right] w(\xi, t) .
\end{aligned}
$$

$$
M_{0}(\xi) \leqq 2 w\left(\xi, t_{0}\right) \quad \text { and } \quad M_{1}(\xi) \leqq 2 w\left(\xi, t_{1}\right) .
$$

To show the first part, we suppose $M_{0}$ occurs at $t=t, t_{0}<t \leqq t_{1}$. Then a straightforward computation shows that

$$
M_{0}(\xi) \leqq 2 \exp \left[-\gamma\left(\bar{t}-t_{0}\right)\right] w\left(\xi, t_{0}\right) \leqq 2 w\left(\xi, t_{0}\right) \text { by using (4). }
$$

Similarly, using (5), if $M_{1}$ occurs at $t=t^{\prime}, t_{0} \leqq t^{\prime}<t_{1}$, one sees that

$$
M_{1}(\xi) \leqq 2 \exp \left[-\gamma\left(t_{1}-t^{\prime}\right)\right] w\left(\xi, t_{1}\right) \leqq 2 w\left(\xi, t_{1}\right) .
$$

Formulas (6) state that

and

$$
\exp \left[-\gamma\left(t-t_{0}\right)\right] w(\xi, t) \leqq 2 w\left(\xi, t_{0}\right)
$$

and thus

$$
\exp \left[-\gamma\left(t_{1}-t\right)\right] w(\xi, t) \leqq 2 w\left(\xi, t_{1}\right)
$$

$$
e^{-\beta(t, \xi)}|\tilde{u}(\xi, t)| \leqq 2 \exp \left[2 C_{0}|\xi|^{j}\left(t-t_{0}\right)\right]\left|\tilde{u}\left(\xi, t_{0}\right)\right|
$$

and

(8) $e^{-\beta(t, \xi)}|\tilde{u}(\xi, t)| \leqq 2 \exp \left[2 C_{0}|\xi|^{j}\left(t_{1}-t\right)\right] \exp \left[-\beta\left(t_{1}, \xi\right)\right]\left|\tilde{u}\left(\xi, t_{1}\right)\right|$ for $-\infty<t_{0} \leqq t \leqq t_{1}<\infty$.

Next we use the fact that $|\xi|^{j} \leqq \delta|\xi|^{k}+C_{\delta}$ for $|\xi|>1$ and any $\delta>0$, where $C_{\delta}=\delta^{-j /(k-j)}$. Then (7) and (8) become

$$
|\tilde{u}(\xi, t)| \leqq 2 \exp \left[2 C_{0}\left(t-t_{0}\right)\left(\delta|\xi|^{k}+C_{\delta}\right)+\beta(t, \xi)\right]\left|\tilde{u}\left(\xi, t_{0}\right)\right|
$$

and

$$
\begin{array}{r}
|\tilde{u}(\xi, t)| \leqq 2 \exp \left[2 C_{0}\left(t_{1}-t\right)\left(\delta|\xi|^{k}+C_{\delta}\right)+\beta(t, \xi)-\beta\left(t_{1}, \xi\right)\right]\left|u\left(\xi, t_{1}\right)\right| \\
\text { for any } \delta>0 \text { and } t \in\left[t_{0}, t_{1}\right]
\end{array}
$$


We restrict our consideration to those $t$ satisfying $t_{0}+\varepsilon_{1}=\tau_{0} \leqq t \leqq \tau_{1}=$ $t_{1}-\varepsilon_{1}$. Since $b_{k} \geqq 0, \beta_{0}(t, \xi)=|\xi|^{-k} \beta(t, \xi)$ is the principal symbol of a peudo-differential operator of order zero and is monotone increasing in $t$ for each $\xi$ fixed $(|\xi|>1)$. Then we set $\eta(t, \xi)=\left[\beta_{0}(t, \xi)+2 C_{0} \delta\left(t-t_{0}\right)\right]|\xi|^{k}$, which is homogeneous of degree $k$ in $\xi$ for $|\xi|>1$, and choose

$$
\delta<\frac{\varepsilon^{\prime}}{2 C_{0}} \min _{t \in\left[\tau_{0}, \tau_{1}\right]} \inf _{|\xi|>1} \frac{\beta_{0}(t, \xi)}{t-t_{0}}, \quad \text { where } \varepsilon^{\prime} \leqq \frac{\beta_{0}(t, \xi)}{\beta_{0}\left(t_{1}, \xi\right)} \leqq 1-\varepsilon^{\prime}
$$

in $\left[\tau_{0}, \tau_{1}\right]$. This can be done because $\beta_{0}$ is the principal symbol of a pseudo-differential operator of order zero.

Then on $\left[\tau_{0}, \tau_{1}\right]$ and for $|\xi|>1$,

$$
\frac{\beta_{0}(t, \xi)}{\beta_{0}\left(t_{1}, \xi\right)+2 C_{0} \delta\left(t_{1}-t_{0}\right)}>\frac{\beta_{0}(t, \xi)}{\beta_{0}\left(t_{1}, \xi\right)} \frac{1}{1+\varepsilon^{\prime}}=\frac{1}{1+\varepsilon^{\prime}} \frac{\beta(t, \xi)}{\beta\left(t_{1}, \xi\right)}
$$

and

$$
\frac{\beta_{0}(t, \xi)+2 C_{0} \delta\left(t-t_{0}\right)}{\beta_{0}\left(t_{1}, \xi\right)}>\frac{\beta_{0}(t, \xi)}{\beta_{0}\left(t_{1}, \xi\right)}\left(1+\varepsilon^{\prime}\right)=\left(1+\varepsilon^{\prime}\right) \frac{\beta(t, \xi)}{\beta\left(t_{1}, \xi\right)} .
$$

Thus,

$$
0<\varepsilon^{\prime} /\left(1+\varepsilon^{\prime}\right)<\eta(t, \xi) / \eta\left(t_{1}, \xi\right)<\left(1+\varepsilon^{\prime}\right)\left(1-\varepsilon^{\prime}\right)<1
$$

for $|\xi|>1$ and $\tau_{0} \leqq t \leqq \tau_{1}$. Formulas (9) and (10) become

$$
|\tilde{u}(\xi, t)| \leqq N_{0} e^{\eta(t, \xi)}\left|\tilde{u}\left(\xi, t_{0}\right)\right|
$$

and

$$
|\tilde{u}(\xi, t)| \leqq N_{0} \exp \left[\eta(t, \xi)-\eta\left(t_{1}, \xi\right)\right]\left|\tilde{u}\left(\xi, t_{1}\right)\right|
$$

for $|\xi|>1$ and $\tau_{0} \leqq t \leqq \tau_{1}$, where $N_{0}=2 \exp \left[2 C_{0}\left(t_{1}-t_{0}\right) C_{\delta}\right]$ and $\eta(t, \xi)$ is the principal symbol of a pseudo-differential operator of order $k$.

Next we set $\alpha(t, \xi)=\eta(t, \xi) / \eta\left(t_{1}, \xi\right)$ for $|\xi|>1$, raise (11) and (12) to the $1-\alpha(t, \xi)$ and $\alpha(t, \xi)$ powers respectively, and multiply the two formulas together. Hence

$$
|\tilde{u}(\xi, t)| \leqq N_{0}\left|\tilde{u}\left(\xi, t_{0}\right)\right|^{1-\alpha(t, \xi)}\left|\tilde{u}\left(\xi, t_{1}\right)\right|^{\alpha(t, \xi)} \text { for }|\xi|>1 .
$$

Note that $0 \leqq \alpha(t, \xi) \nearrow 1$ for $\xi$ fixed, since $\eta(t, \xi)$ is monotone increasing in $t$. If $\alpha$ were independent of $\xi$ we could square the terms in (13), integrate for $|\xi|>1$ and get the relationship we want by using Hölder's inequality. However, by exploiting the facts that $\alpha$ is homogeneous of degree $k$ in $|\xi|$ for $|\xi|>1$ and that there exists $\varepsilon=\varepsilon(t)>0$ such that $\varepsilon \leqq \alpha(t, \xi) \leqq 1-\varepsilon$ for $t$ fixed, we can use an extension of Hölder's inequality (Lemma 3 below). 
This tells us that, for any fixed $t$ in $\left(t_{0}, t_{1}\right)$,

$$
\begin{aligned}
\int_{|\xi|>1}|\tilde{u}(\xi, t)|^{2} d \xi \leqq & \left(\int_{|\xi|>1}\left|\tilde{u}\left(\xi, t_{0}\right)\right|^{2} d \xi\right)^{1-\varepsilon}\left(\int_{|\xi|>1}\left|\tilde{u}\left(\xi, t_{1}\right)\right|^{2} d \xi\right)^{\varepsilon} \\
& +\left(\int_{|\xi|>1}\left|\tilde{u}\left(\xi, t_{0}\right)\right|^{2} d \xi\right)^{2}\left(\int_{|\xi|<1}\left|\tilde{u}\left(\xi, t_{1}\right)\right|^{2} d \xi\right)^{1-\varepsilon} \\
\leqq & \left\|\tilde{u}\left(\cdot, t_{0}\right)\right\|^{2(1-\varepsilon)}\left\|\tilde{u}\left(\cdot, t_{1}\right)\right\|^{2 \varepsilon}+\left\|\tilde{u}\left(\cdot, t_{0}\right)\right\|^{2 \varepsilon}\left\|\tilde{u}\left(\cdot, t_{1}\right)\right\|^{2(1-\varepsilon)} .
\end{aligned}
$$

We may also write

$$
\begin{aligned}
\int_{|\xi| \leqslant 1}|\tilde{u}(\xi, t)|^{2} d \xi & \\
& \leqq C \int_{|\xi| \leqq 1}\left|e^{\eta(t, \xi)} \tilde{u}\left(\xi, t_{0}\right)\right|^{2(1-\varepsilon)}\left|e^{\eta(t, \xi)-\eta\left(t_{1}, \xi\right)} \tilde{u}\left(\xi, t_{1}\right)\right|^{2 \varepsilon} d \xi,
\end{aligned}
$$

since $g$ is bounded on $\{|\xi| \leqq 1\}$. In the compact set $\{|\xi| \leqq 1\}$ the exponentials are bounded, so the integrals make sense. Then

$$
\begin{aligned}
\int_{|\xi| \leqq 1}|\tilde{u}(\xi, t)|^{2} d \xi & \leqq C(\eta, \varepsilon) \int_{|\xi| \leqq 1}\left|\tilde{u}\left(\xi, t_{0}\right)\right|^{2(1-\varepsilon)}\left|\tilde{u}\left(\xi, t_{1}\right)\right|^{2 \varepsilon} d \xi \\
& \leqq C\left(\eta, \varepsilon_{1}\right)\left(\int_{|\xi| \leqq 1}\left|\tilde{u}\left(\xi, t_{0}\right)\right|^{2} d \xi\right)^{1-\varepsilon}\left(\int_{|\xi| \leqq 1}\left|\tilde{u}\left(\xi, t_{1}\right)\right|^{2} d \xi\right)^{\varepsilon} \\
& \leqq C\left(\eta, \varepsilon_{1}\right)\left\|\tilde{u}\left(\cdot, t_{0}\right)\right\|^{2(1-\varepsilon)}\left\|\tilde{u}\left(\cdot, t_{1}\right)\right\|^{2 \varepsilon},
\end{aligned}
$$

as we can use the standard Hölder inequality here, with $p=1 /(1-\varepsilon)$, $q=1 / \varepsilon$. We add (14) and (15) to get

$$
\begin{aligned}
\|\tilde{u}(\cdot, t)\|^{2} \leqq(C(\eta, \varepsilon)+1)\left[\left\|\tilde{u}\left(\cdot, t_{0}\right)\right\|^{2(1-\varepsilon)}\right. & \left\|\tilde{u}\left(\cdot, t_{1}\right)\right\|^{2 \varepsilon} \\
& \left.+\left\|\tilde{u}\left(\cdot, t_{0}\right)\right\|^{2 \varepsilon}\left\|\tilde{u}\left(\cdot, t_{1}\right)\right\|^{2(1-\varepsilon)}\right] .
\end{aligned}
$$

Set $C=C(\eta, \varepsilon)+1$. Then, by applying Parseval's theorem, we obtain the desired inequality.

If $\left[t_{0}+\varepsilon_{1}, t_{1}-\varepsilon_{1}\right]$ is any compact subset of $\left(t_{0}, t_{1}\right)$, then $\varepsilon=\varepsilon\left(\varepsilon_{1}, \eta\right)$ can be found so that $\varepsilon \leqq \alpha(t, \xi) \leqq 1-\varepsilon$ on $\left[t_{0}+\varepsilon_{1}, t_{1}-\varepsilon_{1}\right]$, and the constant in the inequality will become $\max _{t \in\left[t_{0}+\varepsilon_{1}, t_{1}-\varepsilon_{1}\right]}(C(\eta, \varepsilon)+1)$ in this case.

Q.E.D.

It remains to state and prove Lemma 3, which is of independent interest since it is an extension of Hölder's inequality.

LeMma 3. Let $f, g$ be in $L^{2}(y)$ and $0<\varepsilon \leqq \gamma(y) \leqq 1-\varepsilon<1$. Then

$$
\int|f|^{2 \gamma(y)}|g|^{2\left(1-\gamma^{(j(y))}\right.} d y \leqq\|f\|^{2(1-\varepsilon)}\|g\|^{2 \varepsilon}+\|f\|^{2 \varepsilon}\|g\|^{2(1-\varepsilon)}
$$

where $\|=\| \quad \|_{L^{2}(y)}$. 
Note that if $\gamma(y) \equiv \gamma_{0}=$ constant, then the ordinary Hölder inequality implies that

$$
\int|f|^{2 \gamma_{0}}|g|^{2\left(1-\gamma_{0}\right)} d y \leqq\|f\|^{2 \gamma_{0}}\|g\|^{2\left(1-\gamma_{0}\right)}
$$

so that by allowing $\gamma$ to vary, the bound becomes the sum of the bounds in Hölder's inequality for $\gamma_{0}=\varepsilon$ and $1-\varepsilon$ respectively.

Proof of Lemma 3. The proof hinges on the pointwise inequality

$$
\delta^{\gamma(y)} \eta^{1-\gamma(y)} \leqq \max \left(\delta^{\varepsilon} \eta^{1-\varepsilon}, \delta^{1-\varepsilon} \eta^{\varepsilon}\right) \text { for } \gamma \text { as above, }
$$

and any nonnegative constants $\delta$ and $\eta$ as above. For, once we have this inequality, then $\delta^{\gamma(y)} \eta^{1-\gamma(y)} \leqq \delta^{\varepsilon} \eta^{1-\varepsilon}+\delta^{1-\varepsilon} \eta^{\varepsilon}$; so that if we set $f(y)=\delta$, $g(y)=\eta$ and integrate over all $y$, the ordinary Hölder inequality with $p=1 / \varepsilon, q=1 /(1-\varepsilon)$ yields the result. Thus, we must only verify (16). Suppose $\delta^{\varepsilon} \eta^{1-\varepsilon} \geqq \delta^{1-\varepsilon} \eta^{\varepsilon}$. Then $\eta^{1-2 \varepsilon} \geqq \delta^{1-2 \varepsilon}$; i.e., $(\eta / \delta)^{1-2 \varepsilon} \geqq 1$, and thus $\eta / \delta \geqq 1$. (If $\varepsilon=\frac{1}{2}$, this procedure is unnecessary.) So

$$
\begin{aligned}
\delta^{\gamma(y)} \eta^{1-\gamma(y)} & \leqq \delta^{\varepsilon} \eta^{1-\varepsilon} \delta^{\gamma(y)-\varepsilon} \eta^{\varepsilon-\gamma(y)}=\delta^{\varepsilon} \eta^{1-\varepsilon}(\delta / \eta)^{\gamma(y)-\varepsilon} \\
& <\delta^{\varepsilon} \eta^{1-\varepsilon}=\max \left(\delta^{\varepsilon} \eta^{1-\varepsilon}, \delta^{1-\varepsilon} \eta^{\varepsilon}\right) .
\end{aligned}
$$

The proof for $\delta^{1-\varepsilon} \eta^{\varepsilon} \geqq \delta^{\varepsilon} \eta^{1-\varepsilon}$ is similar. Q.E.D.

REMARK. If $b=b_{k}$ and is independent of $t$, then

$$
\alpha(t, \xi)=\frac{b_{k}(\xi)\left(t-t_{0}\right)}{b_{k}(\xi)\left(t_{1}-t_{0}\right)}=\frac{t-t_{0}}{t_{1}-t_{0}}
$$

is independent of $\xi$. Hence the result in this case is

$$
\begin{aligned}
\|u(t)\| \leqq C\left(\left\|u\left(t_{0}\right)\right\|^{\left(t_{1}-t\right) /\left(t_{1}-t_{0}\right)}\left\|u\left(t_{1}\right)\right\|^{\left(t-t_{0}\right) /\left(t_{1}-t_{0}\right)}\right. & \\
& \left.+\left\|u\left(t_{0}\right)\right\|^{\left(t-t_{0}\right) /\left(t_{1}-t_{0}\right)}\left\|u\left(t_{1}\right)\right\|^{\left(t_{1}-t\right) /\left(t_{1}-t_{0}\right)}\right) .
\end{aligned}
$$

\section{BIBLIOGRAPHY}

1. A. Calderón, Uniqueness in the Cauchy problem for partial differential equations, Amer. J. Math. 80 (1958), 16-36. MR 21 \#3675.

2. Existence and uniqueness theorems for systems of partial differential equations, Proc. Sympos. Fluid Dynamics and Appl. Math. (University of Maryland, 1961), Gordon and Breach, New York, 1962, pp. 147-195. MR 27 \#6010.

3. B. Malgrange, Unicité du problème de Cauchy. Division des distributions, Séminaire Schwartz 1959/60, Faculté des Sci., Paris, 1960, §§8-11. MR 23 \#A2275.

4. L. Nirenberg, Pseudo-differential operators, Proc. Sympos. Pure Math., vol. 16 Amer. Math. Soc., Providence, R.I., 1970. MR 42 \#5108. 
5. L. Nirenberg and F. Treves, On local solvability of linear partial differential equations. II. Sufficient conditions, Comm. Pire Appl. Math. 23 (1970), 459-509; correction, ibid. 24 (1971), 279-288. MR 41 \#! J64b.

6. - Remarks on the solvability of line $r$ equations of evolution, Proc. Sympos. on Evolution Equations, Istituto di Alta Materiatica, Rome 1970 (to appear).

7. M. Strauss, Uniqueness and norm convevity for the Cauchy problem, Thesis, New York University, 1971.

Department of Mathematics, TeXas Tech University, Lubbock, Texas 79409 